\title{
INDUCTIVE LIMITS OF $A$-CONVEX ALGEBRAS
}

\author{
ALLAN C. COCHRAN
}

\begin{abstract}
The ideas of inductive limits and bornological spaces are extended to $A$-convex algebras. The usual locally convex linear space characterization of bornological spaces has an appropriate analog for $A$-convex algebras. The results here have a strong connection to locally $m$-convex algebras ( $A$-convexity generalizes $m$ convexity) and some additional information relating to $m$-convex algebras is given. Comparisons of various inductive limits are obtained including results which insure that these limits coincide. Finally, $A$-bornological algebras are introduced and studied. It is shown that the property of being $A$-bornological is preserved with respect to several general constructions.
\end{abstract}

1. Warner [10] studied inductive limits of normed algebras relating these limits to locally $m$-convex algebras. In this note inductive limits of $A$ normed algebras (separate continuity of multiplication) are studied, with the results related to $A$-convex algebras ([4], [5], [6]). Appropriate analogies to the standard results in linear space theory are given. The fact that the set of all $A$-convex topologies on a given algebra $A$ form a complete lattice yields the existence of inductive limits in the category of $A$ convex algebras. The construction of an associated locally $m$-convex topology from a given $A$-convex topology, see [6], allows a detailed comparison between Warner's results and those in the $A$-convex case. Examples are given to illustrate various results. The general setting established by Warner [11] is used to develop the $A$-convex case. Bornological algebras are defined in the context of $A$-convexity ( $A$-bounded, $A$-bornological, etc.) and the standard results of the linear space setting are obtained in this context. It is shown that bornological algebras are preserved under various constructions. The author is indebted to the referee for several helpful suggestions in revising this note.

2. Introduction. This section is concerned with some basic results about $A$-convex algebras. The main proofs and notation may be found in ([4], [5], [6]). All algebras considered in this note are assumed to be locally

Received by the editors May 8, 1972.

AMS (MOS) subject classifications (1970). Primary 46H05, 46H15.

Key words and phrases. A-convex algebra, $m$-convex algebra, bornological space, normed algebra, inductive limit of algebras, Mackey topology. 
convex (algebras over $\boldsymbol{R}$ and $\boldsymbol{C}$ which have a locally convex linear structure and separately continuous multiplication). A subset $V$ of an algebra $E$ is called $A$-convex if $V$ is absolutely convex, absorbing and for each $x$ in $E$, $V$ absorbs $x \cdot V$ and $V \cdot x$. An $A$-convex algebra is an algebra $E$ together with a linear topology on $E$ whose neighborhood system at zero has a basis of $A$-convex sets. Equivalently [4] an $A$-convex algebra is an algebra with a topology defined via a family of $A$-convex seminorms $(p(x y) \leqq$ $M_{x} p(y)$ and $p(y x) \leqq N_{x} p(y)$ hold for all $y$ where $M_{x}$ and $N_{x}$ are constants depending only on $x$ and $p$ ). $A$-convexity is preserved with respect to taking products, subspaces, and quotients. Each $A$-convex algebra can be embedded in an $A$-convex algebra with identity.

Examples [4], [6] of $A$-convex algebras include the locally $m$-convex algebras and $A$-normed algebras (normed linear space with separately continuous multiplication). Another source of examples is given by an algebra $M(A)$ of multipliers on a commutative Banach algebra (without order) $A$. For details see [8]. The strict topology $\beta$ on $M(A)$ is defined by the $A$ convex seminorms,

$$
p_{f}(T)=\|T f\|, \quad T \in M(A),
$$

where $f \in A$. The strict topology due to Buck [3] can be realized as a special case and provide examples of complete $A$-convex algebras which are not locally $m$-convex [4], [6].

Let $\mathscr{A}(E)$ denote the collection of all $A$-convex topologies on an algebra $E$ and let $\mathscr{M}(E)$ denote all locally $m$-convex topologies on $E$. Each of these sets is partially ordered by inclusion and $\mathscr{M}(E)$ is a complete lattice [10].

Proposition 1. The partially ordered set $\mathscr{A}(E)$ is a complete lattice whose largest element is generated by the set of all A-convex subsets of $E$.

Proof. The topology $\mathscr{S}=\{\varnothing, E\}$ is $A$-convex and is the least element of $\mathscr{A}(E)$. Since the finite intersection of $A$-convex sets is $A$-convex, the supremum of an arbitrary nonempty family of $A$-convex topologies is again $A$-convex. Hence $\mathscr{A}(E)$ is a complete lattice.

Warner [10] has shown that for $A \subset E$, the smallest idempotent ( $V$ is idempotent if $V \cdot V \subset V$ ) set containing $A$ is $A_{1}=\bigcup\left\{A^{n}: n=1,2, \cdots\right\}$. Let $A$ * denote the balanced, convex hull of $A_{1}$ for a given set $A$. Let $\tau \in \mathscr{A}(E)$ and let $\mathscr{N}$ denote the neighborhood filter at zero. Then $m(\mathscr{N})=$ $\left\{N^{*}: N \in \mathscr{N}\right\}$ is the neighborhood filter at zero for a locally $m$-convex topology $m(\tau)$ on $E$. We will call $m(\tau)$ the associated locally m-convex topology of $\tau$. This association is used in [6] to show that the associated locally $m$-convex topology for the strict topology $\beta$ is always the compactopen. 
Proposition 2. The map $m: \mathscr{A}(E) \rightarrow \mathscr{M}(E)$ described above is an idempotent map onto $\mathscr{M}(E)$. The topology $m(\tau)$ is the finest locally m-convex topology coarser than $\tau$. Hence, for any algebra $E$, the associated locally $m$-convex topology on E for the strongest A-convex topology is the strongest locally m-convex topology on $E$.

3. Inductive limits. The general setting given by Warner [11] is used here to describe inductive limits of $A$-convex algebras. Michael [7] and Warner [10] have given basic results for locally $m$-convex algebras. Fundamental relationships among the usual linear inductive limit and these two classes of algebras are given. (For notation and definitions see [11].)

Let $\mathscr{L}$ denote the structure on a linear space $E$ consisting of all nonempty convex, balanced subsets of $E ; \mathscr{M}$ the structure on an algebra $E$ consisting of all scalar multiples of all nonempty convex, balanced, idempotent subsets of $E$; and $\mathscr{A}$ the structure on an algebra $E$ of all nonempty convex, balanced subsets $V$ of $E$ which absorb $x \cdot V$ and $V \cdot x$ for all $x$ in the subalgebra generated by $V$. Then $\mathscr{M} \subseteq \mathscr{A} \subseteq \mathscr{L}$ for any algebra $E$. Note that the $A$-convex subsets of $E$ are precisely the elements of $\mathscr{A}$ which are absorbing and the $A$-convex topologies on $E$ are precisely those which are compatible with $A$. The algebraic homomorphisms of $E$ to an algebra $F$ are both $\mathscr{A}$ - and $\mathscr{M}$-structure homomorphisms but not conversely. This introduces the notions of inductive limits, bornological space, bornivorous set, bornological homomorphism into the class of $A$-convex algebras via the structure $\mathscr{A}$ [11]. Existence of inductive limits of $A$-convex algebras follows from Proposition 1 (also from [11]). We denote by $\left\{E_{\alpha}, g_{\alpha} ; \Gamma\right\}$ a family of $A$-convex algebras $E_{\alpha}$ (topology $\tau_{\alpha}$ ) with homomorphisms $g_{\alpha}$ of $E_{\alpha}$ into $E, E$ an algebra, and $\alpha \in \Gamma$, a fixed index set. The inductive limit topology with respect to the structure $\mathscr{A}$ on $E$ will be denoted by $\mathscr{A}\left(\left\{E_{\alpha}, g_{\alpha} ; \Gamma\right\}\right)$, the inductive limit topology with respect to the structure $\mathscr{L}$ will be denoted by $\mathscr{L}\left(\left\{E_{\alpha}, g_{\alpha} ; \Gamma\right\}\right)$ and if each $\tau_{\alpha}$ is locally $m$-convex then $\mathscr{M}\left(\left\{E_{\alpha}, g_{\alpha} ; \Gamma\right\}\right)$ will denote the topology with respect to $\mathscr{M}$. The neighborhood filters at zero will be denoted by $A_{0}, L_{0}$, and $M_{0}$ respectively. By applying Proposition 7 of Warner [11], we have

Proposition 3. Let $\left\{E_{\alpha}, g_{\alpha} ; \Gamma\right\}$ be an inductive family. Then an element $V$ of $\mathscr{A}(\mathscr{M}, \mathscr{L})$ is in $A_{0}\left(M_{0}, L_{0}\right)$ if and only if $V$ is absorbing and $g_{\alpha}^{-1}(V)$ is a $\tau_{\alpha}$-neighborhood of zero for each $\alpha$. If $f$ is any structure homomorphism from $E$ into any structured locally convex space then $f$ is continuous if and only if $f \circ g_{\alpha}$ is continuous for each $\alpha$.

Proposition 4. Let $\left\{E_{\alpha}, g_{\alpha} ; \Gamma\right\}$ be an inductive family on $E$ such that each $E_{\alpha}$ is locally m-convex. Then

$$
\mathscr{M}\left(\left\{E_{\alpha}, g_{\alpha} ; \Gamma\right\}\right) \leqq \mathscr{A}\left(\left\{E_{\alpha}, g_{\alpha} ; \Gamma\right\}\right) \leqq \mathscr{L}\left(\left\{E_{\alpha}, g_{\alpha}: \Gamma\right\}\right) .
$$


Warner [10] and Michael [7] give conditions, in the locally $m$-convex case, which insure that $\mathscr{M}\left(\left\{E_{\alpha}, g_{\alpha} ; \Gamma\right\}\right)=\mathscr{L}\left(\left\{E_{\alpha}, g_{\alpha} ; \Gamma\right\}\right)$. Proposition 4 shows that each of these theorems insure that all three limits mentioned here coincide. For example, a result due to Warner [10] gives the following: If $E$ is an algebra, with $\left\{E_{n}: n=1,2, \cdots\right\}$ an increasing sequence of ideals in $E$ whose union is $E$, and if $\tau_{n}$ is a locally $m$-convex topology on $E_{n}$ for each $n$ such that $\tau_{n+1} \mid E_{n}=\tau_{n}$ then the three limits agree if $\mathscr{M}\left\{E_{n}, i_{n}\right\}$ induces $\tau_{n}$ on each $E_{n}$.

Proposition 5. If $E$ and $F$ each have an A-convex topology and $g$ is a continuous homomorphism then $g$ is continuous with respect to the associated m-convex topologies.

THEOREM 1. Let $\left\{E_{\alpha}, g_{\alpha} ; \Gamma\right\}$ be an inductive family on $E$. Then $m\left(\mathscr{A}\left(\left\{E_{\alpha}, g_{\alpha} ; \Gamma\right\}\right)\right)=\mathscr{M}\left(\left\{m\left(E_{\alpha}\right), g_{\alpha} ; \Gamma\right\}\right)$ where $m\left(E_{\alpha}\right)$ denotes the associated m-convex space $\left(E_{\alpha}, m\left(\tau_{\alpha}\right)\right)$.

Proof. By Proposition 4 and Proposition 5, $m\left(\mathscr{A}\left(E_{\alpha}\right)\right) \leqq \mathscr{M}\left\{m\left(E_{\alpha}\right)\right\}$. If $H$ is in $\mathscr{M}_{0}\left\{m\left(E_{\alpha}\right)\right\}$ then for each $\alpha \in \Gamma, g_{\alpha}^{-1}(H) \supset V_{\alpha}$ for $V_{\alpha}$ in $m\left(\mathscr{N}_{\alpha}\right)$. By definition of $m\left(\mathscr{N}_{\alpha}\right)$,

$$
V_{\alpha} \supset \bigcup\left\{W^{n}: W \in \mathscr{N}_{\alpha}, W \text { an } A \text {-convex set, } n=1,2, \cdots\right\} \text {. }
$$

Hence $g_{\alpha}^{-1}(H) \supset W$ so $g_{\alpha}^{-1}(H)$ is in $\mathscr{N}_{\alpha}$ for each $\alpha \in \Gamma$. Thus $H$ is an element of $A_{0}\left\{E_{\alpha}\right\}$ so $M_{0}\left\{m\left(E_{\alpha}\right)\right\} \geqq A_{0}\left\{E_{\alpha}\right\}$. But $m\left(\mathscr{A}\left\{E_{\alpha}\right\}\right)$ determines the finest $m$ convex topology coarser than $\mathscr{A}\left\{E_{\alpha}\right\}$ (Proposition 2) and $\mathscr{M}\left\{m\left(E_{\alpha}\right)\right\}$ is locally $m$-convex so $m\left(\mathscr{A}\left\{E_{\alpha}\right\}\right) \geqq \mathscr{M}\left(m\left\{E_{\alpha}\right\}\right)$ and equality follows.

Corollary 1. If each $E_{\alpha}$ is locally m-convex and each $g_{\alpha}$ is one-to-one and onto then $\mathscr{A}\left(\left\{E_{\alpha}, g_{\alpha} ; \Gamma\right\}\right)=\mathscr{M}\left(\left\{E_{\alpha}, g_{\alpha} ; \Gamma\right\}\right)$.

Theorem 1 gives the fundamental comparison theorem and a useful criterion for checking the agreement of $\mathscr{A}\left\{E_{\alpha}, g_{\alpha} ; \Gamma\right\}$ and $\mathscr{M}\left\{E_{\alpha}, g_{\alpha} ; \Gamma\right\}$ in case each $E_{\alpha}$ is locally $m$-convex. Namely, these two limits agree if and only if $m(\mathscr{A})=\mathscr{M}$. The next result gives another condition for agreement.

THEOREM 2. Let $(E,\|\|)$ be an $A$-normed algebra and $\left\{E_{\alpha}, i_{\alpha}: \alpha \in \Gamma\right\}$ an inductive family on $E$ such that each $E_{\alpha}$ is a subalgebra of $E, i_{\alpha}$ the inclusion map and $\bigcup\left\{E_{\alpha}: \alpha \in \Gamma\right\}=E$. Then $\mathscr{A}\left(\left\{E_{\alpha}, i_{\alpha} ; \Gamma\right\}\right)=\mathscr{L}\left(\left\{E_{\alpha}, i_{\alpha} ; \Gamma\right\}\right)$.

Proof. By Proposition 4, $\mathscr{L}\left(\left\{E_{\alpha}, i_{\alpha} ; \Gamma\right\}\right) \geqq \mathscr{A}\left(\left\{E_{\alpha}, i_{\alpha} ; \Gamma\right\}\right)$. Let $V$ be an absolutely convex absorbing set with $V$ in $\mathscr{L}\left\{E_{\alpha}\right\}$. Then for each $\alpha \in \Gamma$ there exists $\varepsilon>0$ such that

$$
W_{\alpha}=\left\{x \in E_{\alpha}:\|x\|<\varepsilon\right\} \subset V \cap E_{\alpha} .
$$

Let $W=\bigcup\left\{W_{\alpha}: \alpha \in \Gamma\right\}$. Then $W \subset V$ and $W$ is balanced and absorbing 
(since $\bigcup E_{\alpha}=E$ ). For $x \in E$, there is a constant $N_{x}$ such that

$$
\|x y\| \leqq N_{x}\|y\| \quad \text { for all } y \text { in } E .
$$

Hence $x W \subset N_{x} W$. Similarly there exists $M_{x}$ such that $W x \subset M_{x} W$. Then the convex hull of $W, W^{c}$, is an $A$-convex set with $W^{c} \subset V$. Clearly $W^{c}$ is in $\mathscr{A}\left\{E_{\alpha}\right\}$ so $V$ is in $\mathscr{A}\left\{E_{\alpha}\right\}$. Hence $\mathscr{A}\left(\left\{E_{\alpha}, i_{\alpha} ; \Gamma\right\}\right) \geqq \mathscr{L}\left(\left\{E_{\alpha}, i_{\alpha}: \Gamma\right\}\right)$.

If $E$ is the direct sum of $\left\{E_{n}: n=1,2, \cdots\right\}$ where each $E_{n}$ is a locally $m$-convex algebra, then all three limits coincide. However, for a general family of $m$-convex algebras $\left\{E_{\alpha}\right\}, \mathscr{A}\left\{E_{\alpha}\right\}$ is not necessarily the same as $\mathscr{H}\left\{E_{\alpha}\right\}$.

4. $A$-bornological algebras. In this section further evidence of the utility of $A$-convex algebras is given. An analog to the standard theorem of locally convex linear spaces about bornological spaces is obtained. Warner [10] has given an extension ( $i$-bornological) to locally $m$-convex algebras. As mentioned in the previous section, there are natural definitions available for the "bornological" concept in the $A$-convex algebra case via the structure $\mathscr{A}$. These give a useful analogy to Warner's development of the locally $m$-convex case in [10]. A subset $B$ of an $A$-convex algebra $E$ is called $A$-bounded if there exists an $A$-convex bounded set $S$ and a scalar $\lambda>0$ with $\lambda B \subset S$. If $E$ is an $A$-convex algebra and $B$ is an absorbing set which is $i$-bounded then $B$ is $A$-bounded. However, the unit ball in an $A$-normed algebra (with multiplication not jointly continuous) is $A$ bounded but not $i$-bounded. If $E$ is locally $m$-convex, then the $A$-bounded sets and the $i$-bounded sets coincide. The class $\mathscr{B}$ of all $A$-bounded subsets of an $A$-convex algebra $E$ form the "bounded sets" in Warner's scheme. For the particular case of an $A$-convex algebra with its $A$-bounded subsets, Warner's treatment gives the following definitions. If $E$ and $F$ are $A$ convex algebras and $f: E \rightarrow F$, then $f$ is $A$-bounded if $f(B)$ is bounded whenever $B$ is $A$-bounded. The algebra $E$ is $A$-bornological if every $A$ bounded homomorphism of $E$ into an $A$-convex algebra $F$ is continuous. An $A$-convex subset $B$ of $E$ is $A$-bornivorous if $B$ absorbs every $A$-bounded set. The scalar multiples, subsets, closed absolutely convex hulls, finite products, and finite unions of $A$-bounded sets are $A$-bounded. If $E$ is $A$ normed, then the bounded sets and the $A$-bounded sets coincide. Hence, if $E$ is an $A$-normed algebra, every $A$-bounded homomorphism is bounded and therefore continuous.

An $A$-convex algebra is pointwise $A$-bounded (pAb) if every singleton is an $A$-bounded set. Any $A$-normed algebra is pAb. The usual requirement that $E=\bigcup\left\{g_{\alpha}\left(E_{\alpha}\right): \alpha \in \Gamma\right\}$ for an inductive limit space $E$ of $\left\{E_{\alpha}, g_{\alpha} ; \Gamma\right\}$ must be dropped to get the standard theorem of bornological spaces for $A$ convex algebras. If $E$ is a bornological $A$-convex algebra such that every bounded set is $A$-bounded then $E$ is $A$-bornological and pAb. 
Let $(E, \tau)$ be an $A$-convex algebra. It follows from results in [11] that the family of all $A$-bornivorous subsets of $E$ is a fundamental neighborhood system of zero for an $A$-convex topology $\tau^{*} ; \tau^{*} \geqq \tau$ and the bounded sets for $\tau$ are identical to the bounded sets for $\tau^{*} ;\left(E, \tau^{*}\right)$ is $A$-bornological; the space $(E, \tau)$ is $A$-bornological if and only if $\tau=\tau^{*}$; and if $f$ is any homomorphism of $E$ into an $A$-convex algebra $F$, then $f$ is $A$-bounded if and only if it is continuous with respect to $\tau^{*}$. All of these statements can be easily proven directly using standard techniques developed in the linear or $m$-convex case. The strongest $A$-convex topology on $E$ is clearly $A$ bornological. The next theorem gives an analog to a theorem about bornological locally convex linear spaces and also $i$-bornological locally $m$-convex algebras.

TheOREM 3. Let $(E, \tau)$ be an A-bornological algebra. Then $(E, \tau)$ is the inductive limit of $A$-normed algebras $\left\{E_{B}: B \in \mathscr{B}\right\}$ for $\mathscr{B}$ the family of all closed $A$-bounded $A$-convex subsets of $E$. The following results are obtained:

(1) $E=\bigcup\left\{E_{B}: B \in \mathscr{B}\right\}$ iff $E$ is $p A b$.

(2) If $E$ is sequentially complete then so is each $E_{I 3}$.

(3) If $E$ is advertibly complete then each $E_{B}$ is advertibly complete.

Proof. For each $B \in \mathscr{B}$, let $E_{B}=\{\lambda x: \lambda \in C, x \in B\}$ and $p_{B}(y)=$ $\inf \{\lambda>0: y \in \lambda B\}$. Then $p_{B}$ is an $A$-convex norm on $E_{B}$ which determines an $A$-convex topology $\tau_{B}$. The inductive limit of $\left\{\left(E_{B}, \tau_{B}\right), i_{B} ; \mathscr{B}\right\}$ is $(E, \tau), i_{B}$ denoting the identity map from $E_{B}$ to $E$; clearly $\tau_{B} \geqq \tau \mid E_{B}$, and if $V$ is any $A$-convex subset of $E$ such that $V \cap E_{B}$ is a neighborhood of zero in $E_{B}$ for all $B \in \mathscr{B}$ then $V \cap E_{B}$ absorbs $B$. Thus $V$ is $A$-bornivorous and consequently $V$ is in $\tau$. This concludes the proof of the first part. The remaining parts are proved in an analogous manner to the corresponding proofs for locally $m$-convex algebras.

A result of this theorem is that a sequentially complete $A$-bornological algebra is the inductive limit of complete $A$-normed algebras and hence of Banach algebras.

THEOREM 4. Let $(E, \tau)$ be a separated A-convex algebra. Then the following are equivalent:

(1) $(E, \tau)$ is the inductive limit of $A$-normed algebras.

(2) $(E, \tau)$ is A-bornological.

(3) No stronger A-convex topology has the same A-bounded sets.

(4) Every A-bornivorous set is a neighborhood of zero.

(5) The strongest A-convex topology compatible with the duality $E$, $(E, \tau)^{\prime}$, is $\tau$ and every A-bornological linear form is continuous.

(6) Every A-bornological linear map of $E$ into an $A$-convex algebra $F$ is continuous. 
By applying the results in [11] and some easy calculations to the $A$ convex case, the following results can be obtained.

THEOREM 5. (1) The inductive limit $\left(E, A\left(\left\{E_{\alpha}, g_{\alpha} ; \Gamma\right\}\right)\right)$ of $A$-bornological spaces $E_{\alpha}$ is A-bornological.

(2) If $H$ is a closed ideal of an A-bornological space $E$, then $E / H$ is A-bornological.

(3) If $f$ is a continuous open homomorphism of $E$ onto $F$, then $F$ is $A$ bornological.

(4) The topological direct sum of A-bornological algebras is A-bornological.

(5) The finite product of $A$-convex algebras is $A$-bornological if and only if each component space is A-bornological.

If $(E, \tau)$ is $A$-bornological, then it is the limit of $A$-normed algebras $\left\{E_{\alpha}: \alpha \in \Gamma\right\}$ by Theorem 4 . If the associated space $m\left(E_{\alpha}\right)$ is normable for each $\alpha \in \Gamma$ then $(E, m(\tau))$ is $i$-bornological by Theorem 1 and the locally $m$-convex analog to Theorem 4 .

Warner [10], [11] proved that the statement "arbitrary products of $i$-bornological $m$-convex algebras (with identity) are $i$-bornological" is equivalent to set theoretic statements. If $E$ is a locally $m$-convex algebra and $V$ is an $A$-bornivorous set then $V$ is $i$-bornivorous (simply use the idempotent envelope and $m$-convexity). From this observation it follows that if the arbitrary products of $A$-bornological algebras are $A$-bornological, then Axiom $U$ and statements (U1)-(U8) of [10] hold. The converse is still an open question. That is, is the $A$-bornologicity of arbitrary products of $A$-bornological algebras equivalent to the statements of [10]? If the answer is affirmative, then yet another class can be added in Proposition 13 of $[11]$.

\section{REFERENCES}

1. G. R. Allan, A spectral theory for locally convex algebras, Proc. London Math. Soc. (3) 15 (1965), 399-421. MR 31 \#619.

2. R. F. Arens, A generalization of normed rings, Pacific J. Math. 2 (1952), 455-471. MR 14, 482.

3. R. C. Buck, Bounded continuous functions on a locally compact space, Michigan Math. J. 5 (1968), 95-104.

4. A. C. Cochran, C. R. Williams and R. Keown, On a class of topological algebras, Pacific J. Math. 34 (1970), 17-25. MR 42 \#8278.

5. A. C. Cochran, Weak-A-convex algebras, Proc. Amer. Math. Soc. 26 (1970), 73-77. MR 41 \#7435.

6. - Topological algebras and Mackey topologies, Proc. Amer. Math. Soc. 30 (1971), 115-119.

7. E. A. Michael, Locally multiplicative-convex topological algebras, Mem. Amer. Math. Soc. No. 11 (1952). MR 14, 482. 
8. J. Wang, Multipliers of commutative Banach algebras, Pacific J. Math. 11 (1961), 1131-1149. MR 25 \#1462.

9. S. Warner, Weak locally multiplicatively-convex algebras, Pacific J. Math. 5 (1955) 1025-1032. MR 17, 876.

10. - Inductive limits of normed algebras, Trans. Amer. Math. Soc. 82 (1956), 190-216. MR 18, 52.

11. - Bornological structures, Illinois J. Math. 4 (1960), 231-245. MR 22 \#1807.

Department of Mathematics, University of ARKansas, Fayetteville, Arkansas 72701

Current address: Department of Mathematics, Duke University, Durham, North Carolina 27706 